\title{
Viability of Fog Methodologies in IoT aware Smart Grid Architectures
}

\author{
Md. Muzakkir Hussain ${ }^{1}$, Mohammad Saad Alam², M.M. Sufyan Beg ${ }^{1}$, Mohammad \\ Asaad $^{2}$ \\ ${ }^{1}$ Department of Computer Engineering, ZHCET, AMU \\ md.muzakkirezhcet.ac.in \\ mmsbeg@cs.berkeley.edu \\ ${ }^{2}$ Department of Electrical Engineering, ZHCET, AMU \\ saad.alamezhcet.ac.in \\ mohammad. asaadezhcet. ac. in
}

\begin{abstract}
Contemporary Smart Grid (SG) systems are enticed by smart devices and entities due to unfolded developments in intelligent transportation technologies (ITT). The SG ecosystem, when introduced to Internet of Things (IoT) makes every object active and brings them online. However, the traditional cloud deployments look puerile to meet the analytics and computational exigencies for such dynamic subsystems. Starting with highlighting the mission critical requirements of an idealized SG infrastructure, this work proposes an edge centered FOG (From cOre to edGe) computing model primarily focused to realize the processing and computational objectives of SG. The motive of the work is to comprehend the applicability of fog computing algorithms to interplay with the core centered cloud computing support, thus enabling to come up with a new breed of real-time and latency free utilities. Further, for demonstrating the feasibility of the proposed framework, a comparative optimization framework is proposed that captures the monetary expenses due to the power consumption, latency and emission issues in both cloud based as well as fog commuting frameworks. Finally, the suitability and viability of fog computing approaches are demonstrated through its comparative results of the metrics with that of traditional data center or cloud computing approach. Results clearly demonstrate the superiority of FOG computing over its cloud counterpart.
\end{abstract}

Keywords: Smart Grid (SG), Internet of Things (IoT), Fog computing, Cloud computing, Advanced Metering Infrastructures (AMI), software defined networking $(S D N)$.

\section{Introduction}

There are relentless economic as well as environmental arguments in the academia, industries, R\&Ds and legislative bodies for the overhaul of the contemporary power grid comprehended by a full Smart Grid rollout [1]. The latter integrates green cum renewable energy production utilities, robust power monitoring schemes, adapts and evolves with the consumption behavior and requirements. However, the unique feature that overlays on the heap of a SG amenities is connectivity and real-time analytics [2]. The recent advancements in information and communication infrastructures in general and Internet of Things (IoT) utilities in specific redefine the notion of "SMART" in current SG architectures. This work outlines the fog 
computing paradigm and examines its primacy over the cloud computing counterpart that became ubiquitous in fulfilling the computational and analytics needs of a reliable, robust, resilient and sustainable SG.

The notion of smartness has been introduced into the contemporary SG architectures where the local nodes will be leveraged with computational capabilities. They will no longer remains a "thing" rather will be transformed into active computing nodes or "objects". Every component of SG network whether it is at the generation, transmission or service level, will act as active nodes in the entire transportation web. They are now called object in the sense that they will be having attributes, gateways, states etc. The whole transportation system can be encapsulated as a network of active nodes having deterministic state transitions. This is achieved through the notion of internet of thing (IoT). IoT will ensure real-time transport of information to and from the utilities, smart grid system and other components in the power system and charging infrastructure in a way that the current as well as future needs of these entities along with dedicated business engagement can be determined [3].

Such technologies are enabled by the recent developments in RFID, smart sensors, communication standards, and Internet protocols[4]. The basic principle is to have an environment where smart sensors collaborate directly with the "objects" without human involvement aiming to deliver multitudes of applications and services [5], [6]. It is a consensus belief by industries as well as research giants that down the line, in near future IoT will emerge as a technology enabler for smart transport, X2X (where $\mathrm{X}$ may be any of but not necessarily same from entities like Vehicles, Grids, Homes, Micro-grid etc.) data and energy exchange topologies, optimal renewable integration and intelligent charging infrastructures [7].

However, it is obvious that in the IoT architecture the population of connected entities will overshoot the current growth drift and will jeopardize the normal computing configurations [8]. This will in turn cause an exponential escalation in data generation, handling of which is key task to ensure viable implementation any data aware infrastructure. Connecting the objects through edge networks and technologies such as Wireless Sensor Networks (WSN), Zigbee, bluetooth, RFID, WiFi, 3G, and 4G etc. will increase the complexity of underlying communication architectures. Efficient and robust data analytics setup that can establish a real-time cum intelligent decision making atmosphere at every edge services becomes the need of hour. An exhaustive review of existing control paradigms reveal the presence centralized coordination strategies such as cloud computing, grid computing etc [8],[9]. However the service demands of IoT architecture reflect that there needs computing schemes that can execute locally at the edge itself. The prevalent cloud models are not intended to handle the seven unprecedented V's (Volume, Velocity, Variety, Variability, Veracity, Visualization and Value) in the data generated by IoT architectures and coupling the whole universe of "things" or "objects" directly to the cloud is nearly unfeasible [10]. Fog computing approaches seem to be the preeminent preference for computations at the extreme edges such as vehicles, roadways, charging station etc [10]-[13].

However installation of fogs (mini data centers) everywhere across the edges of the networks and entities may not be cost productive. The infrastructure demands varying 
levels of services which in turn have specified QoS requirements. Transporting terapeta bytes of data from millions of edge devices to the central cloud in real-time is quite infeasible and even unessential, as a significant percentage of data are passive and don't contribute to any decision making process. Furthermore, there exist several tasks that don't even entail storage, processing and analytics at cloud scale. Such requirements motivate the need of a hybrid control architecture where the mining and analytics activities are intelligently dispersed.

The smart grid applications require location aware geodistributed intelligency in services such as metering information updates, power thefts, distribution outages, network intrusions etc, and require prompt and reflex actions to evolve and organize according to the adversaries. However, the current smart grid is under immense pressure owing to its sullen response to the abovementioned computational demands. Also, due to its fragility concerns in SG control and coordination sub-systems, repercussions of power outages, resiliency and reliability issues are growing ever more serious. Upgrading to a computationally smarter, reliable and resilient grid has escalated from being a desirable vision, to an urgent imperative. Here we itemize few but not the least, of some of the mission critical requirements of an ideal SG infrastructure plus the sombre experiences encountered while going for pure cloud computing deployment.

\subsection{Support for scalable real-time services:}

The need of real-time analytics and decisions is being emerged as the need for the hour to carry up the timing requirements of mission critical SG utilities [14]. Even if some servers' fiascos occur, the system should heal itself with just graceful degradation in latency services. The current cloud models support for SGs can provide rapid response mechanisms but adversaries still pose threats to responsiveness.

\subsection{Support for scalable, consistency guaranteed, fault-tolerant services:}

Consistency for cloud-hosted utilities is a broad term associated with ACID (Atomicity, Consistency, Isolation and Durability) guarantees, support for state machine replication, virtual synchrony, and support for only limited count of node failures [1]. Today's smart grid cloud infrastructures often "embrace inconsistency", thus implementing consistency preserving computational structures constitute a nascent thrust domain for the research \& development sector.

\subsection{Privacy and security:}

The woeful protection services of current cloud deployments often stimulate the cloud vendors to recapitulate their security management folks to "not be evil". Stern efforts are in progress across the power system and transportation communities to come up with SG cloud utilities and platforms leveraged with robust protective contrivances where the stakeholders could entrust the storage of sensitive and critical data even under concurrent share and access architectures [15], [16]. 


\subsection{Highly Assured Connectivity:}

Added with power outages, the smart grid consumers also experience intermittence in data connectivity. Projects for establishing mechanisms dedicated to support secured multipath data routing from user edges to cloud services are on headway. Critical components of the future smart grid applications demand better quality of service $(\mathrm{QoS})$ and quality of experience $(\mathrm{QoE})$ from the data routing backbone that underlie the cloud-hosted utilities.

\subsection{Need for risk management modules:}

Switching from traditional power grid to multi-tenant SG subsystems introduce substantial risks to power sector, an issue that need to get fixed in inception phase. The penetration of autonomous EVs into modern road transport manifolds such concerns. The EVs are becoming a basin for multi-dimensional data production, an asset if mishandled, may befool the execution of whole systems. Moreover, the data generated due to Cloud-IoT integrated transportation telematics coupled with advanced metering infrastructures (AMI) can prove to be harmful to its stakeholders, specifically for privacy and security [16]. Thus, it's an earnest need for the stakeholders to be assured with stringent protection protocols and be inert from the vulnerabilities. Such scenario necessitates incorporating robust risk analysis procedures that will evaluate and quantify the computational and business risks that persist in such critical infrastructures. Selection followed by implementation of proper risk analysis paradigms is itself a full-fledged realm to dwell on. Risks perceived to be minor in inception phase, later elicits tougher public concerns. Though the "pay-forusage" protocols of cloud computing business models are efficient in satisfying the bulky analytics and computational tasks, the bliss transforms into worries when the applications demand null-latency services and when the data stream chokes the bandwidth restricted communication buses. The emerging wave IoT based transportation telematics can prove potentially astonishing in fulfilling the mobility requirements of contemporary smart grid architectures.

Motivated by the above mentioned mission critical Smart Grid requirements, the pitfalls associated with current cloud computing infrastructures to meet such needs, and having the assumption that the smart grid community is not in a position to reinvent a remotely owned Internet infrastructure or to develop computing platforms and elements from scratch, this work presents a fog computing framework whose principle underlie on offloading the time and resource critical operations From corre to ed $\mathbf{G}$ e. The argument here is not to cannibalize the existing cloud support for SG, but to comprehend the applicability of fog computing algorithms to interplay with the core centered cloud computing support leveraged with a new breed of real-time and latency free utilities.

\section{Fog Architecture for Smart Grid}

The work presents a three schema computing architecture where the significant portions of smart grid control and computations are non-trivially hybridized alongside the cloud computing support. The objective is to overcome the disruption caused by 
the development of IoT utilities where the control, storage, networking and computational needs are actively proliferated across the edges or end-points.

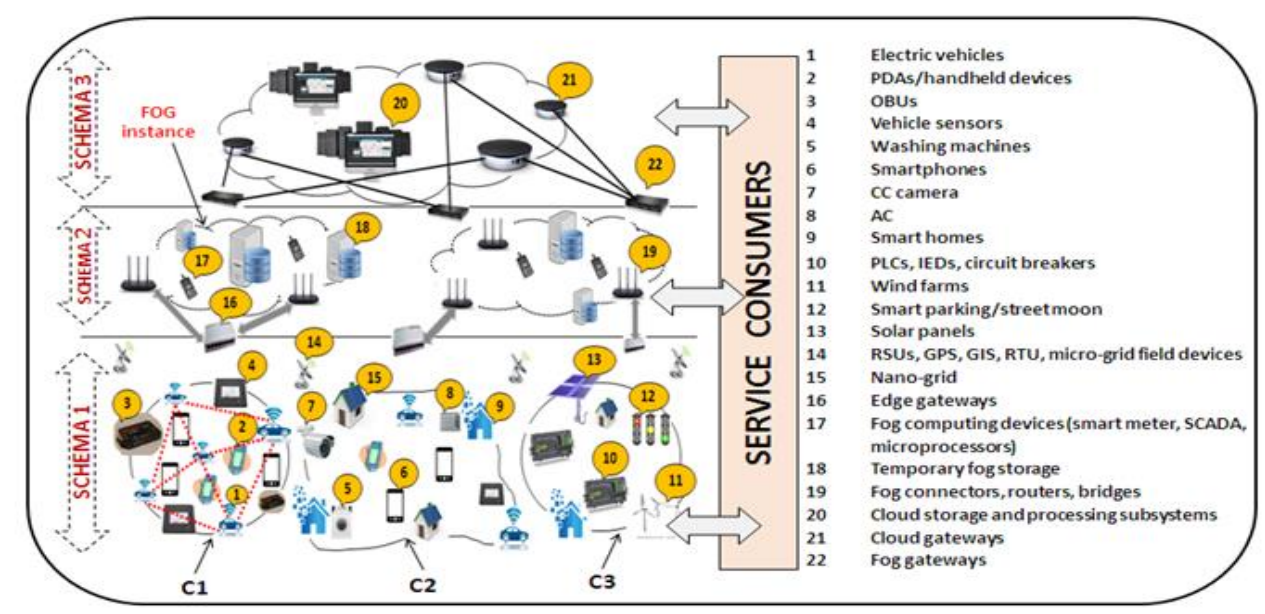

Fig. 1: Topology of FOG computing paradigm in a Smart Grid

The lowermost schema namely physical schema or data generator layer primarily comprises of a wide range of smart IoT enabled devices which come within the SG domain. For simplicity, the entities are abstracted into logical clusters of applications, directly or indirectly influenced by the expediency of SG operations.

The first cluster (C1) represents vehicular applications where the intelligent vehicles are arranged to form vehicular fogs. The existing transportation telematics support such as cellular telephony, on-board sensors (OBS), roadside units (RSU), and smart wearable devices will uncover the computational as well as networking capabilities latent in the underutilized vehicular resources. The notion is to employ the underutilized vehicular resources into communicational and analytics use, where a collaborative multitude of end-user clients or near-user edge devices carry out communication and computation, based on better utilization of individual storage, communication and computational resources of each vehicle [5]. Similarly, similar presence of clusters $(\mathrm{C} 2)$ could also be traced in smart home networks that have a noteworthy contribution in consistent operations of the backend SG support. The intelligent IoT equipped home gadgets such as washing machines, AC, freezes, parking lots, CC camera etc, are also potentially active to provide storage, analysis and computational support for satisfying the prompt and local decision making services. The third but not the least, cluster C3 depicts similar structure that can be constituted by utilities involved at the extreme ends of a SG infrastructure viz. micronano grid, PLCs, automated circuit breakers and other entities associated to diverse range of SG generation, transmission and distribution services. 
The smart nodes within such clusters sense and cultivate the heterogeneous physical attributes and transmit it to the upper layers through dedicated edge gateways. However, the whole or a portion of data generated within these physical clusters are accumulated at the interim across access points such as global positioning (GPS), GIS, road-side units (RSU),remote terminal units (RTU), intelligent electronic devices (IED), phasor data concentrators (PDC), and other field arrays.

The next tier constitutes the fog computing layer comprising of intelligent fog devices such as SCADA, smart meters, routers, switches high-end proxy servers, intelligent agent and commodity hardware etc, having peculiar ability of storage, computation and packet routing. The software defined networking (SDN) assembles the physical clusters to form virtualized inter cluster private networks (ICPN) that route the generated data to the fog devices spanned across the fog computing layer. The fog devices and its corresponding utilities form geographically distributed virtual computing snapshots or instances that are mapped to lower layer devices in order serve the processing and computing demands of SG.

\section{Networking and Operation Model}

For demonstrating the feasibility of a customized fog computing architecture in smart grid sub-systems proposed in section 2, the work develops metrics that correlates the performance of fog computing services to that of traditional cloud computing paradigms. The geo-distributed micro datacenters in a typical fog model performs a significant proportion of local computations on the data produced by data generators at the schema 1. However, the devices are leveraged with distributed intelligence, in that depending upon the degree of services criticality and the types of data, the righteous decision of whether to offload the data to the cloud or to the local micro-data centers can be undertaken. For SG applications, the fog computing framework outperforms its pure cloud counterparts in respect to metrics like power consumption, latency and carbon footprint (emission) etc.

Consider a pilot SG analytics service to be delivered from the three tier fog architecture devised in section II over a 24 hour time horizon. Out of volume of data generated in the whole day, the preprocessing and decision modules deployed in the first tier offloads into the mega datacenters for cloud level analytics while distributes to the micro datacenters for local and instant processing and computations. Further, a significant portion of gets filtered or cutout at the fog computing layer (schema 2) i.e. they don't demand cloud level computations or permanent storage, rest are offloaded to the cloud for historical or less critical analysis operations or into the permanent storage. The uncertainty in the data distributions across multiple schemas is captured in probability factors depicted in figure 2 .

An ideal fog-cloud framework is leveraged with robust inferencing logic and intelligent filtering devices to undertake instant decisions on where to distribute the produced datasets. The objective of the proposed framework is to minimize the cost encountered due to power consumption, latency and emission issues. In case of fog computing approach, an additional cost term needs to be added due to communication among the IoT enabled sensors as well as micro datacenters. 


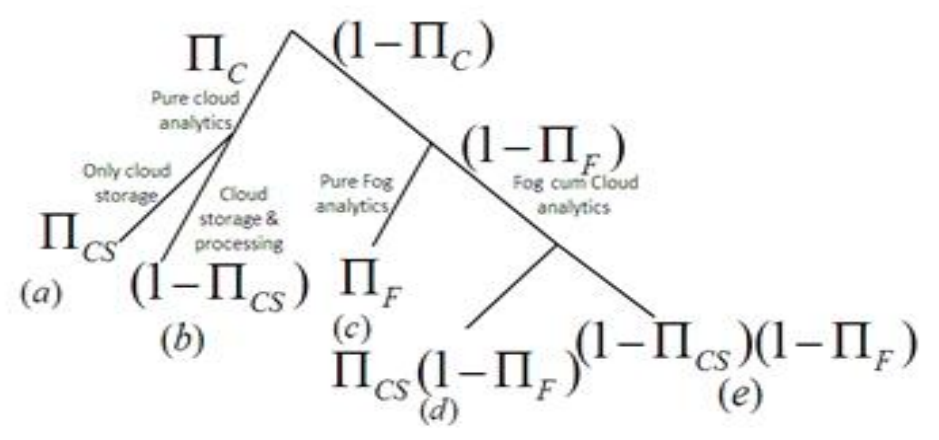

Fig 2: Decision tree for analysis and computation services

\subsection{Cost profile for Generic Cloud processing:}

$$
F_{C}(x)=C_{C}(v)+C_{C}(w)+C_{C}(c)
$$

Where $F_{C}(x), C_{C}(v), C_{C}(w)$ and $C_{C}(c)$ represent the overall cost function, power consumption cost (incurred due to storage and execution of the data at the mega datacenters), cost due to latency terms and the cost due to carbon footprint at the data centers respectively.

$C_{C}(v)=\left[\begin{array}{l}V_{1} \cdot \Pi_{C}\left\{\Pi_{C S} \cdot\left(\alpha_{C S}\right)+\left(1-\Pi_{C S}\right) \cdot\left(\alpha_{C A}\right)\right\}+ \\ \left(1-\Pi_{C}\right) \cdot\left(1-\Pi_{F}\right) \cdot\left(\alpha_{C A}\right) \cdot V_{3}\end{array}\right] \cdot \lambda_{E}$

Where, $\alpha_{C S}, \alpha_{C A}$ and $V_{3}$ represent the power consumed in cloud storage, cloud storage cum processing and amount of data that is migrated to the cloud layer through cloud-fog gateway interface (21-22 in fig 1$). \lambda_{E}((\mathrm{USD} / \mathrm{KWh}))$ is the energy to cost conversion factor.

$C_{C}(w)=\left[\begin{array}{l}\left(\Pi_{C} \cdot \frac{V_{1}}{w_{D C}}\right)+\left(1-\Pi_{C}\right) \cdot\left(1-\Pi_{F}\right) \cdot V_{3} \\ \cdot\left(\frac{1}{w_{D E}}+\frac{1}{w_{E F}}+\frac{1}{w_{F C}}\right)\end{array}\right] \cdot \lambda_{L}$

$\lambda_{L}$ (USD/Minutes) is the delay to cost conversion factor.

$$
C_{C}(c)=\chi \cdot\left\{V_{1} \cdot \Pi_{C}+V_{3} \cdot\left(1-\Pi_{C}\right)\right\} \cdot \lambda_{G}
$$


$\chi$ and $\lambda_{G}$ represents the gas emissions rate from the data centers and emission to cost conversion factor.

\subsection{Cost profile for Fog aware Cloud processing:}

$F_{F}(x)=C_{F}(v)+C_{F}(w)+C_{F}(c)$

Where $F_{F}(x), C_{F}(v), C_{F}(w)$ and $C_{F}(c)$ represent the overall cost function, power consumption cost (incurred due to storage and execution of the data at the micro datacenters), cost due to latency terms and the cost due to carbon footprint at the local data centers respectively.

$C_{F}(v)=\left[\begin{array}{l}\left(V_{2}-V_{3}\right) \cdot\left(1-\Pi_{C}\right) \cdot\left\{\Pi_{F} \cdot\left(\alpha_{F A}\right)+\right. \\ \left(1-\Pi_{C}\right) \cdot\left(1-\Pi_{F}\right) \cdot\left(\alpha_{F S}\right) \cdot V_{3}\end{array}\right] \cdot \lambda_{E}$

$\alpha_{F A}$ and $\alpha_{F S}$ represent the energy consumed in fog processing, fog processing plus cloud storage respectively.

$$
\begin{gathered}
C_{F}(w)=\left[\begin{array}{l}
\left(1-\Pi_{C}\right) \cdot \Pi_{F} \cdot\left(V_{2}-V_{3}\right) \cdot \frac{1}{w_{D E}}+ \\
\left(1-\Pi_{C}\right) \cdot\left(1-\Pi_{F}\right) \cdot V_{3} \cdot\left(\frac{1}{w_{E F}}+\frac{1}{w_{F C}}\right)
\end{array}\right] \cdot \lambda_{L} \\
C_{F}(c)=\chi \cdot\left\{\begin{array}{l}
\left(V_{2}-V_{3}\right) \cdot\left(1-\Pi_{C}\right) \\
+V_{3} \cdot\left(1-\Pi_{C}\right) \cdot\left(1-\Pi_{C}\right)
\end{array}\right\} \cdot \lambda_{G}
\end{gathered}
$$

$w_{D C}, w_{D E}, w_{E F}$ and $w_{F C}$ represent the bandwidth of the channel linking generators to mega datacenters (when data demands pure cloud storage or computations), generators to edge routers, edge routers to fog gateways and fog gateways to cloud gateways respectively.

\subsection{Optimization model:}

In order to assess the viability of proposed fog computing framework, in this subsection a cost optimization model is proposed. The objective is to reveal the fact that, if properly designed, a fog computing framework can circumvent intricacies prevalent in contemporary cloud computing paradigms. The following optimization framework captures the scenario where former outperforms the later in terms overall performance.

Maximize $\left\{\min F_{F}(x)-\min F_{C}(x)\right\}$

Subject to:

$V=V_{1}+V_{2}$ 
$C_{F}(c)<C_{C}(c)$
$C_{F}(w)<C_{C}(w)$

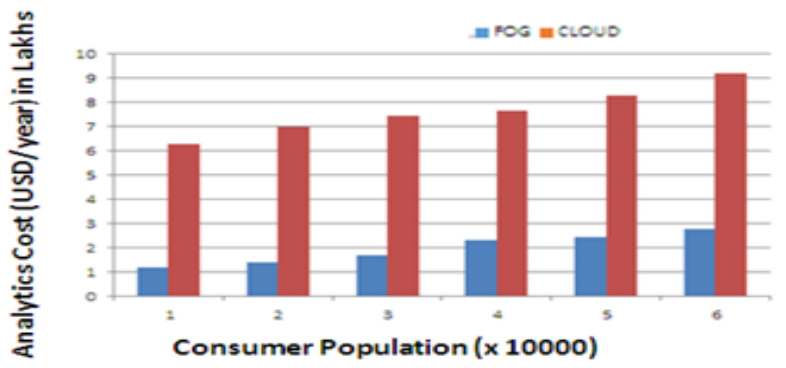

Fig 3. Comparison of per year investment on computations in fog and generic cloud deployment model.

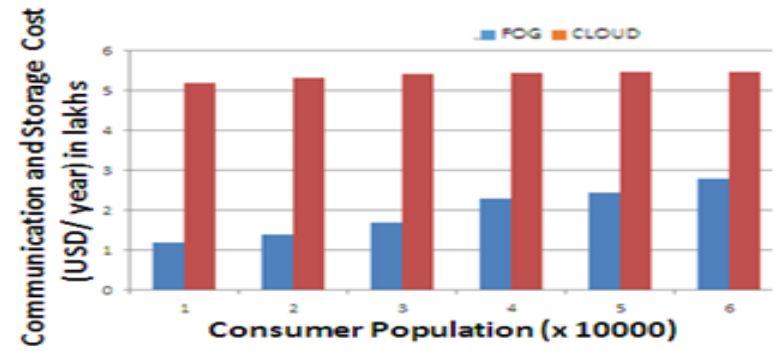

Fig 4. Comparison of per year investment on routing and processing in fog and generic cloud deployment model.

The formulation involves more constraints defining the data consumer-fog node mappings or associations, constraints related to load distribution among the fog processing nodes, constraints describing the coverage range of each fog instances and also constraints which define the overall quality of service (QoS) as well as quality of experience (QoE) of overall execution architecture. Objective function (9) under the aforesaid constraints give rise to a mixed integer non-linear programing (MINLP) formulation. This MINLP problem needs to be first transformed into MILP through linearization techniques. For realizing the viability of proposed framework, the coordinates of eight major data centers across the world are considered. To estimate the IoT users, top 100 cities according to population density are taken and the number user is taken to be proportional to population density. Due to space and time limitations, the authors skipped the implementation details and only presented the results for validation of the proposed model. Figure $3 \& 4$ depicts the respective improvements in the cost incurred in analytics and routing plus storage in for and cloud paradigms. 


\section{Conclusion}

In this paper, an edge centered FOG computing model is proposed for smart grids infrastructures. The work first exposes the inadequacies of pure cloud based SG subsystem to meet the processing and computational requirements and proposes a model that ensures proper distribution and offloading of computations across the cores (cloud) as well as edges. For assessing the viability of the proposed framework, an SG use case is explored. An optimization framework is presented that captures the cost terms associated to power consumption, latency and emission parameters. The viability of the proposed model is demonstrated through comparative results performed on pilot datasets under real world assumptions and constraints. The superiority of fog computing methods is reflected through the results and discussions. A further enhancement to the mentioned framework along with its validation and deployment in real world scenario are under progress and is presumed to demonstrate in future works.

\section{References}

[1] K. Birman, L. Ganesh, and R. van Renesse, "Running Smart Grid Control Software on Cloud Computing Architectures 1 . Introduction: The Evolving Power Grid," Work. Comput. Needs Next Gener. Electr. Grid, pp. 1-28, 2010.

[2] X. Fang, S. Misra, G. Xue, and D. Yang, "Smart Grid - The New and Improved Power Grid :," IEEE Commun. Surv. Tutorials, vol. 14, no. 4, pp. 944-980, 2012.

[3] R. Emilia, R. Emilia, P. G. V. Naranjo, M. Shojafar, and L. Vaca-cardenas, "Big Data Over SmartGrid -A Fog Computing Perspective Big Data Over SmartGrid - A Fog Computing Perspective," no. November, 2016.

[4] A. Al-Fuqaha, M. Guizani, M. Mohammadi, M. Aledhari, and M. Ayyash, "Internet of Things: A Survey on Enabling Technologies, Protocols, and Applications," IEEE Commun. Surv. Tutorials, vol. 17, no. 4, pp. 2347-2376, 2015.

[5] X. Hou, Y. Li, M. Chen, D. Wu, D. Jin, and S. Chen, "Vehicular Fog Computing: A Viewpoint of Vehicles as the Infrastructures," IEEE Trans. Veh. Technol., vol. 65, no. 6, pp. 3860-3873, 2016.

[6] F. Y. Okay and S. Ozdemir, “A Fog Computing Based Smart Grid Model,” vol. 10, no. 6, pp. 16, 2016.

[7] F. Bonomi, R. Milito, J. Zhu, and S. Addepalli, "Fog Computing and Its Role in the Internet of Things," Proc. first Ed. MCC Work. Mob. cloud Comput., pp. 13-16, 2012.

[8] E. van der Zee and H. Scholten, "Big Data and Internet of Things: A Roadmap for Smart Environments," Stud. Comput. Intell., vol. 546, pp. 137-168, 2014.

[9] Z. Xiao, Y. Xiao, and S. Member, "Security and Privacy in Cloud Computing," vol. 15, no. 2, pp. 843-859, 2013.

[10] B. Davis, "The 7 pillars of Big Data," Pet. Rev., no. January, pp. 34-36, 2015.

[11] S. Sarkar, S. Chatterjee, and S. Misra, "Assessment of the suitability of fog computing in the context of internet of things," IEEE Trans. Cloud Comput., vol. PP, no. 99, pp. 1-14, 2015.

[12] I. Stojmenovic and S. Wen, "The Fog Computing Paradigm: Scenarios and Security Issues," Proc. 2014 Fed. Conf. Comput. Sci. Inf. Syst., vol. 2, pp. 1-8, 2014.

[13] B. Varghese, N. Wang, D. S. Nikolopoulos, and R. Buyya, "Feasibility of Fog Computing," 2017.

[14] Cisco Systems, "Fog Computing and the Internet of Things: Extend the Cloud to Where the Things Are," Www.Cisco.Com, p. 6, 2016.

[15] Y. Wang, T. Uehara, and R. Sasaki, "Fog computing: Issues and challenges in security and forensics," Proc. - Int. Comput. Softw. Appl. Conf., vol. 3, pp. 53-59, 2015.

[16] K. Lee, D. Kim, D. Ha, U. Rajput, and H. Oh, "On security and privacy issues of fog computing supported Internet of Things environment," 2015 Int. Conf. Netw. Futur. NOF 2015, 2015.

[17] A. V. Dastjerdi, H. Gupta, R. N. Calheiros, and S. K. Ghosh, "Fog Computing: Principles , Architectures, and Applications," pp. 1-26. 